\title{
Traffic Accident Reconstruction Technology Research
}

\author{
Xiaolong Yang \\ Institute of Computer Science of Sichuan Normal \\ University \\ Chengdu, Sichuan, China \\ E-mail: 1138721953@qq.com
}

Tao Lv

Institute of Computer Science of Sichuan Normal University

Chengdu, Sichuan, China

E-mail: lvtao@163.com

\author{
Ping Li \\ Institute of Physics and Electronic Engineer of Sichuan \\ Normal University \\ Chengdu, Sichuan, China \\ E-mail: 568811843 @qq.com \\ Xuehua Liao \\ Institute of Computer Science of Sichuan Normal \\ University \\ Chengdu, Sichuan, China \\ E-mail: liaoxuehua@163.com
}

\begin{abstract}
Based on the virtual simulation theory, we used three-dimensional modeling software to build modeling road facilities (vehicles, trees, street lights, etc.) for simulating the accident environment, and by using OpenGL technology, achieved reading, displaying and controlling the threedimensional models. This dynamically realized the threedimensional animated simulation of vehicle movement. Simultaneously we have calculated in progress the simulation of vehicle crash with the basic theory of automobile collision, vehicle collision model and the law of conservation of energy and momentum. Finally, we constructed a flexible platform for the simulation experiment. The platform is enabling to add and update road, trees, street lamps and house on the simulation environment dynamically, and has ability to analysis the traffic accident. This could give an assistant to the handling traffic accidents.
\end{abstract}

Keywords-Virtual Reality; Vehicle Crash; OpenGL Technique; Accident Reconstruction

\section{INTRODUCTION}

With the growth of car ownership, road traffic accidents have become a major social problem worldwide, and have brought serious disaster to human life and property safety. Some research institute at home and abroad have done a lot of study on the computer simulation of traffic accidents, including the establish, analysis and optimization of the vehicle collision models and the kinetic models, as well as the study and the optimization of collision detection, mart traffic liability determination after the simulation. Traffic collision type is varied, the environment of collision is complex, collided vehicles' type are also different, most domestic study is about only one specific area or vehicle collision environmental, lack versatility and maneuverability.

This article corrected the shortcomings above, and builds a road traffic accident reconstruction simulation system which is generic. The system use three-dimensional modeling software to simulate things that are involved in traffic accidents environment such as vehicles, trees, street lights and other road infrastructure, and use OpenGL technique to achieve the reading, displaying, and controlling the three-dimensional model, and dynamically build the scene of road and its environment, as well as threedimensional animated simulation of the vehicle. At the same time, the system can draw the road dynamically, load the trees, street lights, and houses etc. for the simulation environment dynamically, and can easily update the simulation vehicle library.

\section{RESEARCH AT HOME AND ABROAD}

There are a lot of research methods and directions in the study of traffic accident reconstruction and simulation. For example, the study of the parameters optimization used in the simulation make the system optimize the input parameters, and then use the optimized parameters to call the optimal collision model and dynamic model to simulate; the research of collision detection, the core of which is the selection and optimization of the algorithm; the discuss of calculation and simplify for car crash. Whatever study method and direction, the computer Virtual reality and 3D animation simulation are important parts.

Many foreign countries have been more complete theory in road traffic simulation and accident reconstruction, and successively developed some application systems for accident reconstruction and analysis, such as the large accident reconstruction software systems SMAC, CRASH and IMPACT which are developed with the financial help of NHTSA. SMAC (Simulation Model of Automobile Collision Program) mainly use the numerical integration of Newton's second law to solve, while CRASH (Calspan Reconstruction of Accident Speed on the Highway Program) used the conservation of energy before and after the collision and the momentum conservation of the translation process to solve; JARI (Japanese Automobile Research Institute) developed J2DACS, Austria developed PCCRASH and HVOSM. PC-CRASH was developed by Dr. H.Steffan to simulate the traffic accidents and is constantly improving in recent years. These systems are based on 
driving and dynamic model, and use two-dimensional or three-dimensional images to show simulation results ${ }^{[3-6]}$.

Much foreign software has lots of input parameters, of which there are some professional parameters, and ordinary users feel difficult to use them. Plus the actual circumstances of each country is different, so we cannot copy foreign software to apply to our country's road safety, we should establish the road safety software in line with China's national reality.

The research institutes in domestic have begun the study of computer simulation, car collisions and other traffic accidents. Tsinghua University, Jilin University and other universities have achieved certain results in this area of the accident dynamic simulation, but the virtual reality technology and other related technologies are still in the initial stage in our country, so we need further development and improvement, therefore the study of accident reconstruction simulation has important significance.

\section{KEY TECHNOLOGY OF ACCIDENT RECONSTRUCTION AND SIMULATION}

\section{A. OpenGL technology}

OpenGL (Open Graphics Library) technique is a library of 3D graphics standard developed by the United States advanced graphics and high-performance computer systems company SGI, which has the function to draw threedimensional graphics. It is dealing with dedicated graphics hardware software interface to support the realization of visualization. OpenGL technique is the best environment for the development of interactive $2 \mathrm{D}$ and $3 \mathrm{D}$ graphics applications. 3D simulation based on OpenGL technique has a wide range of applications, high quality and high performance, especially in superior programming performance, multi-platform, hardware independence, and the network transparency superiority ${ }^{[1-2]}$.

The Accident Reconstruction basic framework of the simulation system is built on the VC single document technology which based on OpenGL technique. The main steps and key technologies are described as follows:

(1) Firstly, in the process of the creation a single document window, we should set the display pixel format and the window's attributes and styles according with the requirements of OpenGL technique.

(2) Secondly, we should get the windows device context table, and then link it with the OpenGL rendering description table.

(3) Thirdly, we should call the OpenGL commands to draw graphics.

(4) At last, we should close the OpenGL graphics window, release the OpenGL rendering described table-RC and Windows device description Table-DC.

\section{B. Car collision detection}

Automobile collision accident experiments in this present study are divided into two types, one is pushing the experiment, and the other is back stepping experiment. In the pushing the experiment, we used the initial speed and collision model to determine whether the collision occurred. If the collision was occurred, we would calculate the postcollision velocities according to the dynamic model, the collision trajectory and the final position, then reproduced the whole process by using the three-dimensional animation. In the other experiment, we calculated the traffic accident both sides' speed according to the termination of the state of the car, the site of ground traces collision position, as well as some other parameters, and use of three-dimensional animation to reproduce. During this process, we could analysis the traffic accident and ascertain the cause of the accident. In the system, using the animation effect is to clear, intuitive reaction of the entire process.

Whether in pushing experiment or in back stepping experiment, we must be on the detection of vehicle collision. In the detection of vehicle collision the high level of realtime was required, such as the collision detection between the two vehicles would complete in a very short of time. But there were contained a lot of objects in a virtual environment, and coupled with the complex shapes of these objects, and therefore it would spent a lot of time to detect collisions between these objects. It is very important to improve simulation authenticity, credibility, and enhance the realism of the virtual environment and immersive with the accurate collision detection, and thus the collision detection is actually bottleneck to construct a virtual environment and simulation system.

In this paper, we described a simplified collision detection model to depict the collision detection, which used in our experiments. In this simplified model, the vehicle is simplified as the smallest rectangle that surrounded the vehicle, so the two vehicles' collision detection is transformed into the problem of intersection of two rectangles.

\section{1) One-dimensional collision model}

For one-dimensional collision, when the distance of two vehicles' center is equal to or less than the distance of the connection center to the front of the two vehicles, we think the two vehicles collided. Reference presented a mathematical model of one-dimensional collision, which showed as in figure 1 and figure 2:

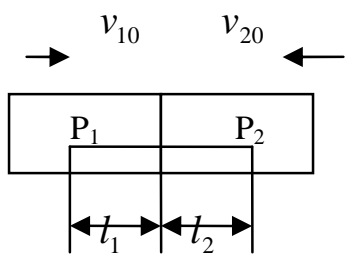

Figure 1. Schematic diagram of head-on collisions 


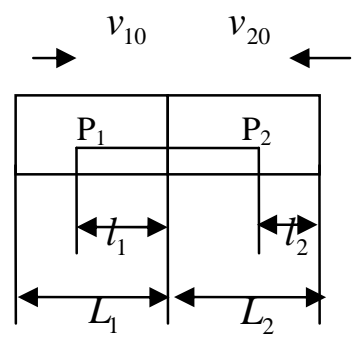

Figure 2. Schematic diagram of rear-end collision

For the head-on collisions, the conditions for the occurrence to determine is $\left|P_{1} P_{2}-l_{1}-l_{2}\right|<\varepsilon$, the $\varepsilon$ is a threshold value, which represents a very small distance and can be determined by the speed and other parameters in the program. Similarly, for the rear-end collisions, the conditions for the occurrence to determine is $\left|P_{1} P_{2}-l_{1}-\left(L_{2}-l_{2}\right)\right|<\varepsilon$.

\section{2) Two-dimensional impact model}

For the two-dimensional collision, the collision is usually occurred by a corner of a car with a particular side of another car, so we can use the relationship between the corner and the edge to determine the collision of two vehicles.

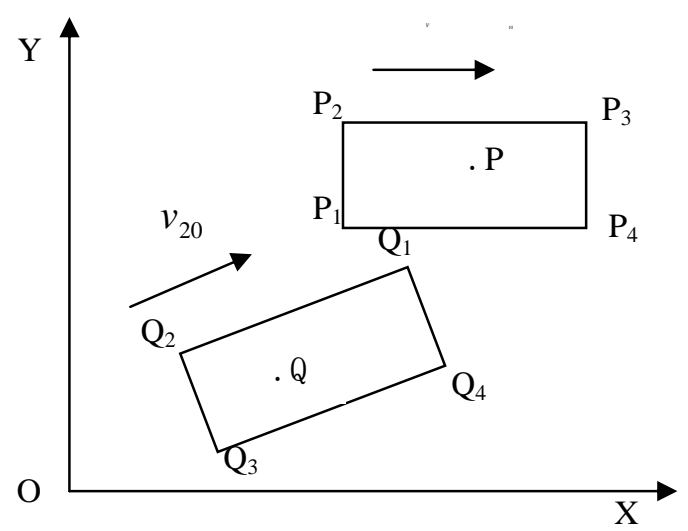

Figure 3. the Schematic diagram of the two-dimensional model for two cars collision

Assuming Q1, which is the front of Q car is collided with P1P4 the side of $\mathrm{P}$ car, then we can know the collision occurred whether or not through determining the relationship between Q1 and P1P4, that is we can use $\left|Q_{1} P_{1}+Q_{2} P_{2}-P_{1} P_{2}\right|<\varepsilon$ to determine the collision occurred whether or not between the front of $\mathrm{Q}$ car and the side of $\mathrm{P}$ car, Because each vehicle's corner has the same probability to collision with the four appearances of another car. Therefore, the four corners of a car collide with another vehicle four sides have 16 kinds of situations, the situation similar to the other car. So for the two-dimensional collisions, we need to consider the situation of the two vehicles collision in a total of 32 possibilities. In each calculate cycle, we must separately determine 32 kinds of collisions which occur whether or not, and can use in the loop programming to realize.

\section{Traffic accidents environment dynamic simulation}

The real scene simulation can give users a sense of realism and immersion. In the pushing experiment, the system should be realize the experimental environment as depicting as possible, and the results can only authentic, the simulation of the various types of data can only be used for analysis, predication of the traffic condition. In the back stepping test, the system should be realizing the experimental environment as realistic as possible, the reproduced simulation results will be reliable, and the reproduction data can be used for auxiliary traffic control department to make traffic accident liability penalty.

Simulations of the scene are divided into traffic accident simulation, relatively static simulation of objects and simulation of the relative motion objects. In the relatively static simulation, such as roads, railings, sidewalks, flowerbeds, street lights, control lights, speed limits, no parking signs, etc., and in the simulation of the relative motion objects, such as car, pedestrians and weather conditions, etc., the reproduction simulation module is the core of the entire system, which described as figure 4 :

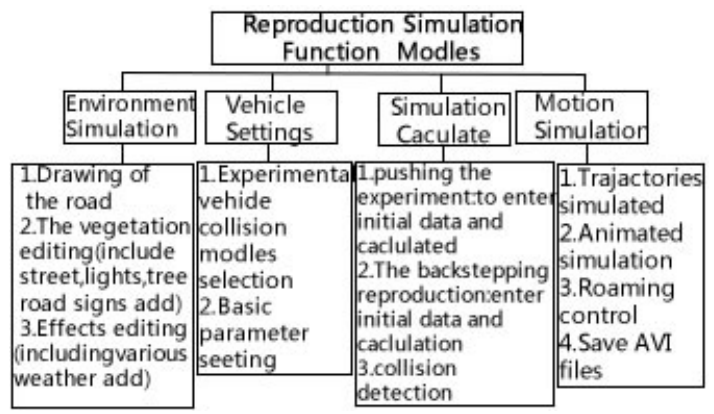

Figure 4. the Simulation Function Structure Diagram

\section{1) Environment simulation function module}

Firstly, according to the traffic accidents environment or test environment we establish a basic simulation scenario by road rendering module function. Secondly, by using coordinates extracted vegetation editing module and the left mouse button, we can rich scenes, add trees, street lights, and road signs and pedestrians scene elements. Finally, we can change the effects editing module parameters to achieve a variety of weather or day and night simulation. By using the audio editing module and the environment simulation module, we can have a kind of comfortable feeling.

The development of environment simulation module is a long-term process of accumulation, in the system we can give the road dynamic rendering, editing of plants, sunny, rainy, fog and daytime and dark days and other special effects. The richness of the scene elements is an effective 
means to increase scene realism and dynamic sense, so the premise does not affect the overall speed of the platform to enrich the scene simulation.

2) Vehicles setting function module

In the system, the user needs to be choosing suitable 3DS model according with the simulated vehicle type, at the same time, some basic parameters of vehicle are entered, such as the mass of the vehicle, the geometry parameters of vehicle. the rotation inertia amount of vehicle and the wheel wheelbase of vehicle information, etc.

\section{3) Simulation Function Module}

The system can either complete the reproduction of the vehicle accidents, and can complete the vehicle collision test of work under certain conditions. Because the two processes are precisely the opposite, only different in the time of the initial input parameters, so it is divided into two small modules, one is pushed experiment module and the other is back stepping reproduction module. But whether in pushed experiment or in back stepping reproduction, the centric position of the vehicle, body angle, angular velocity before and after the collision, car direction, speed, etc. are all important parameters. According to the classification of traffic accidents, the accident simulation modules can be simplified as calculation module, refined as a simple accident, two vehicles one-dimensional collision calculation module, the central collision calculation module and generally two-dimensional collision calculation module.

\section{4) Movement Process Simulation Function Module}

According to the vehicle parameters and the parameters of the initial state of the vehicle, we can calculate the movement process and the collision process, and then can know the movement and termination state of vehicles, so we can simulate the entire trajectory of the motion process, and can play the real-time animation simulation. It is worth noting that the simulation system is simulating the state of the vehicle motion, which includes the simulation of the collision process and non-collision process simulation. In the system, various simulation processes controlled by roaming, can be observed from a different perspective. Finally, we save the simulation animation files as AVI files for later experiments to reference, at the same time, the system will give some parameters of the vehicle, such as the crash position before collision, the vehicle speed, the position after collision, the car speed of traffic accidents etc., it will give some supporting role to response the penalty of traffic accident.

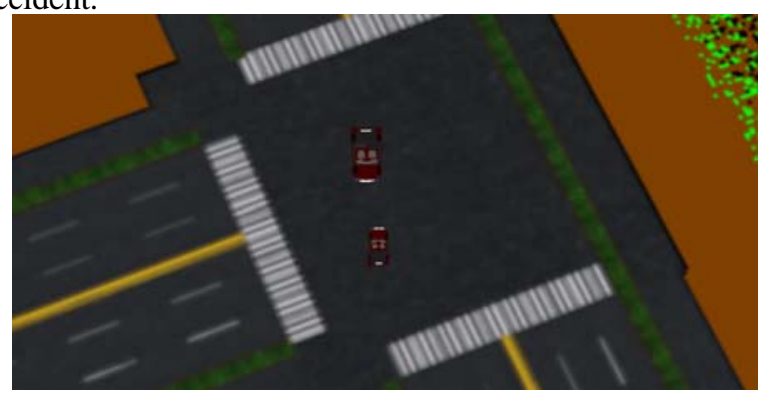

Figure 5. the Simulation Vehicles Traveling Interface

\section{THE REALIZATION OF ACCIDENT RECONSTRUCTION EXPERIMENTAL SIMULATION}

\section{A. Development environment}

The overall system architecture is consists of the following development tools and controls, as well as hardware and software platform:

\section{- Hardware: PC, Windows OS}

- The development tools: VC++6.0 + OpenGL

- Database: MS SQL Server 2005

\section{B. Simulation process}

The overall processes of the traffic accidents experiment simulation system are shown in figure 6:

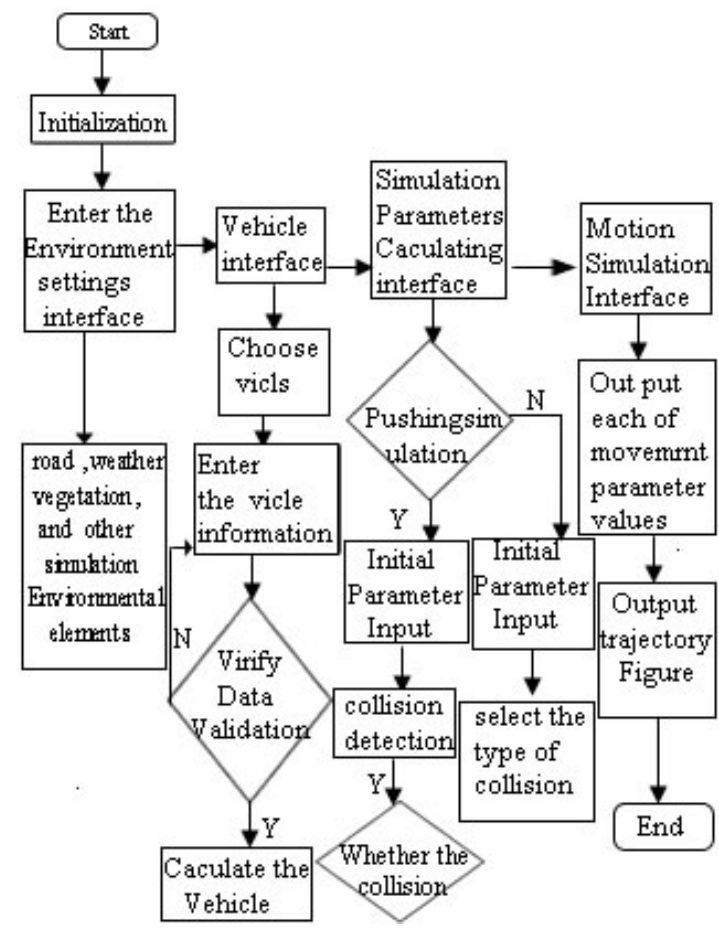

Figure 6. the Overall Flow Chart of the Traffic Accidents Experimental Simulation

There are no excessive demands of the system function models. In model designing, all functions can be arbitrary 3DMAX technology, but for the material aspects, because the program of OpenGL Technique only can read the BMP images which size is $2^{\wedge} \mathrm{n}$ pixel size, so it is required for texture slightly. In addition it should be paid attention to conserve resources during the entire system of the modeling process, and cannot be just for effect and regardless the cost. The following are described the considerations of the system modeling:

(1) The size of the scene is to be consistent with the real size, and the units are to be reasonable. 
(2) In the system there are must be considered in the quantity and the rendering speed of the model.

(3) In order to save resources, we use the OpenGL blanking technology to eliminate redundant facets during the simulation.

(4) The size of the texture must be deal the BMP bitmap which size is $2^{\wedge} \mathrm{n}$ pixel size by using OpenGL technique.

\section{CONCLUSIONS}

Because there have a lot of details problem of the building for reproduction platform and have some complex simulation environment of vehicle collision, so we have only simulated the road and weather environment and have analysis the one-dimensional and two-dimensional collision model of the accident vehicle impact. Finally we have calculated the simulation model of vehicle movement during traffic accident. But we haven't research for the vehicle roll, the collision of automobile with pedestrian, the collision of vehicle with stationary objects and with non-motor vehicle, these studies have being conducted.

\section{REFERENCES}

[1] LIAO Xue-hua, ZHU Zhou-sen, et al. The Study of Traffic Accident Reproduction System, IEEE International Conference on Computer Science and Automation Engineering,2011, 7:367-370.

[2] Xue-hua LIAO, Zhou-sen ZHU, et al. Traffic Accident Reconstruction Technology Research and Simulation Realization. IEEE Symposium on Electrical \& Electronics Engineering, 2012, 7:152-155.

[3] Wei lang, etc. A Study of Computer Simulation System for Reconstructing Car-to-Car Collisions Traffic Accidents[J], China Journal of Highway and Transport, 1996.9(4):105-110.

[4] Li Yi-bing, etc. Application of OpenGL in Traffic Accident Reconstruction[J], Journal of Highway and Transportation Research and Development, 2001.18(14):112-115.

[5] PEI Jian ping, etc. Overview of Impact Models in Accident Reconstruction[J], Journal of Traffic and Transportation Engineering, 2001.12,1(4):75-78.

[6] DING Tong-qiang, etc. Study and Trend of the Technology of Accident Reconstruction[J], Journal of Kunming University of Science and Technology, 2004.6,29(3):102-105.

[7] Zeng Junwu, etc. A Simplified Mathematical model for Collision Detection in Vehicle Driving[J], Computer Simulation, 2000.17(6):13-15. 